COMMENT. Deficits in short-term memory and developmental delays, previously noted in infants and at 4 years of age in children exposed to PCBs in utero, have now been demonstrated in children tested at 11 years of age. PCBs may have a long-term adverse effect on cognitive function, and prenatal exposure to these environmental toxins should be included among potential causes of attention deficit disorders in children.

\title{
PREDICTIVE VALUE OF LEAD SCREENING PRACTICES
}

The prevalence of elevated blood lead levels and the accuracy of a lead screening questionnaire in an urban pediatric population were evaluated in the primary care clinics of 10 community health centers in the city and county of Denver, CO. Of approx 3000 low-income children tested, only $0.3 \%$ had blood lead levels $>20 \mathrm{mcg} / \mathrm{dL}$. The predictive value of the CDC questionnaire was $3 \%$, little better than chance, and the cost of identifying a child with a lead level > $20 \mathrm{mcg} / \mathrm{dL}$ was approx \$5000. (France EK, Gitterman BA, Melinkovich P, Wright RA. The accuracy of a lead questionnaire in predicting elevated pediatric blood lead levels. Arch Pediatr Adolesc Med Sept 1996;150:958-963). (Respond: Eric K France MDCM, Department of Preventive Medicine, Kaiser Permanente, 10350 E Dakota Ave, Denver, CO 80231).

COMMENT. The Editor, Dr Catherine D DeAngelis, notes that "when a survey questionnaire is only slightly better than chance, it's better to take the chance and save the money for the blood tests." The Denver Health and Hospitals chose to screen all low-income children between 12 and 30 months of age and to forgo the use of the questionnaire. Universal screening may be omitted in low-prevalence communities, according to current CDC guidelines.

A recent survey of a nationally representative sample of pediatricians found that $53 \%$ screen all their patients aged 9 to 36 months, $96 \%$ using a blood lead assay. Most of the remainder report screening of high-risk patients only. (Campbell JR, Schaffer SJ, Szilagyi PG et al. Blood lead screening practices among US pediatricians. Pediatrics Sept 1996;98:372-377). In pediatric neurology practice, a blood lead level may be indicated in high-risk children who present with neurodevelopmental delay, ADHD, seizures, or signs of encephalopathy or neuropathy.

\section{CARBAMAZEPINE VS VALPROATE AND COGNITIVE FUNCTION}

Effects of carbamazepine vs valproate on cognitive functioning in patients with previously unmedicated epilepsy were evaluated in a prospective, randomized, double-blind Veterans Affairs multicenter study. Patients with seizures showed deficits relative to a normal control group prior to AED therapy. No significant decline from baseline levels of neuropsychological performance was detected over 6- or 12-month treatment intervals for either drug. Patients with high serum VPA levels (mean, 94 $\mathrm{mcg} / \mathrm{mL}$ ) performed less well than controls on measures of concentration and memory. Subtle compromises of cognitive functioning following treatment with VPA or CBZ were suggested by absence of practice effects. (Prevey ML, Delaney RC, Cramer JA et al. Effect of valproate on cognitive functioning. Comparison with carbamazepine. Arch Neurol Oct 1996;53:1008-1016). (Reprints: Mary L Prevey PhD, Neurology 127, VA Medical Center, West Haven, CT 06516).

COMMENT. Carbamazepine and valproate monotherapies have subtle effects on cognitive functioning, when studies are well controlled. 